\title{
Equilibrium Selection in Interference Management Non-Cooperative Games in Femtocell Networks
}

\author{
Ana Galindo-Serrano ${ }^{1}$, Eitan Altman ${ }^{2}$, and Lorenza Giupponi ${ }^{1}$ \\ ${ }^{1}$ Centre Tecnològic de Telecomunicacions de Catalunya (CTTC) \\ Parc Mediterrani de la Tecnologia, Av. Carl Friedrich Gauss 7, Barcelona,Spain 08860 \\ e-mail: \{ana.maria.galindo,lorenza.giupponi\} @cttc.es \\ ${ }^{2}$ INRIA Sophia Antipolis 2004 route des Lucioles 06902 Sophia Antipolis, France \\ e-mail: eitan.altman@inria.fr
}

\begin{abstract}
In this paper we apply a special class of $n$-person non-cooperative games, which we call Weakly Coupled Constrained Games (WCCG), in femtocell systems to manage the aggregated interference they may generate at macrocell associated users. WCCG have the following structure: the utility of a player depends only on its own assignment and interactions between players appear through extra constraints. Non-cooperative games with common constraints have infinitely many equilibria, we focus on selecting one, the normalized Nash equilibrium, which has some desirable scalable properties related to pricing, establish its uniqueness and compute it in a closed form.

Index Terms-Femtocell system, interference management, non-cooperative game, equilibrium selection, normalized equilibrium.
\end{abstract}

\section{INTRODUCTION}

Femtocells or Home eNodeBs (HeNBs), following the 3rd Generation Partnership Project (3GPP) nomenclature, are small-coverage cellular Base Stations (BSs) to be deployed by end users to provide or improve indoor coverage and enhance the users capacity. HeNBs are one of the components of the recently introduced concept of heterogeneous networks, as part of the Long Term Evolution-Advanced (LTE-A) technology, which proposes to combine different access network technologies to increase the network capcity [6]. HeNBs have two important configuration characteristics, i.e. users access privileges: closed, open and hybrid access and frequency of operation: dedicated band or co-channel operation. In terms of users access privileges, closed HeNBs only can be accessed by those users who belong to its Closed Subscriber Group (CSG). Open HeNBs can give service to any mobile in the network as long as they have available resources. Finally, hybrid HeNBs can be accessed by CSG members as well as non-CSG members. In terms of frequency of operation, in one hand, HeNBs can work in a dedicated band, which avoids the interference with the macrocell system or evolved NodeB (eNB) system, as defined by 3GPP, but goes against the current Cognitive Radio (CR) efficient frequency use trends. On the other hand, HeNBs can perform in co-channel operation, which allows a higher frequency efficiency but introduces a potential aggregated interference problem in the eNB system [17].
HeNBs can be considered as low interfering systems since their required transmission power is low due to the short distance of their communications and also because of the isolation properties of walls. Anyhow, when dense urban deployment models are considered, for the case of closed access and co-channel based HeNBs, aggregated interference problem has to be considered, i.e. multiple HeNBs simultaneously transmitting could cause harmful interference to potentially close macrousers.

In literature, multiple works addressing this timing problem can be found. The Small Cell Forum presented a study regarding interference management in Orthogonal Frequency Division Multiple Access (OFDMA) femtocells [9] and concluded that interference between femtocells and between macro and femtocells remains the most detrimental performance factor in heterogeneous networks. In [5], the authors introduce an uplink capacity analysis and an interference avoidance strategy based on feasible combinations of average number of active macrousers and femtocell BSs per cell-site. Reference [10] proposes a decentralized interference control based on potential games. Some guidelines on spectrum allocation and interference mitigation based on self-configuration and selfoptimization techniques are presented in [12]. A distributed and dynamic carrier assignment method for downlink interference avoidance is proposed in [16]. In [7] a downlink interference management in OFDMA networks, based on distributed gradient descent method is presented and in [4] the authors present an algorithm based on game theory and stochastic approximation to mitigate femto-to-macrocell crosstier interference.

In this paper we consider a situation in which multiple HeNBs coexist with an eNB, both systems based on Long Term Evolution (LTE). We assume HeNBs working in cochannel operation and with closed access privileges, which is the most complex situation in terms of intercell interference. We assume that interference at macrousers coming from other eNBs is negligible, due to the considered 3-cell frequency reuse cluster deployment, as well as the one at HeNB originated from eNBs and neighboring HeNBs, due to the wall penetration losses. We then focuss on fulfilling two restrictions, the first one common to the multiple HeNBs regarding 
the amount of interference they generate at the macrocell users and the second, a local one, regarding the HeNB's total transmitted power. We model the given problem as a noncooperative $n$-person game, where players are the multiple HeNBs facing an optimization problem with restrictions. Each player $i$ in the game is characterized by a set of strategies $S_{i}$ and an utility function. Depending on the relation between this two parameters and other players' strategies, $n$-person games can be classified in three different models, orthogonal, coupled and generalized. $n$-person games with orthogonal constraints, describe those situations where agent's restrictions are independent of other players' strategies. The existence and uniqueness of the equilibrium point in these games was proved by Nash in [13]. Coupled constraints games, are those games where players share a common restriction, therefore the utility and strategies of each player may depend on the strategies of other players. Finally, for the case of generalized constraints, all players do not necessarily share common constraints.

Given the above description of the type of $n$-person games, we focus on coupled constraint games since our players aim is to maximize their individual utility, subject to a common global constraint, the total interference at macrousers. We follow the Generalized Nash Equilibrium Problem (GNEP) natural extension of the standard Nash equilibrium concept proposed by Nash to players sharing common resources or limitations [8]. GNEP is currently widely used in multiple and different fields such as mathematics, economics, engineering, telecommunications, computer science, etc. since it perfectly describes competitive situations in distributed decision making systems. Some routing games with capacity constraints have this structure, where the set $S_{i}$ of available strategies to player $i$ are those for which the sum of flows in each link cannot exceed the link's capacity. It is an extension of the constrained satisfaction games recently introduced by Perlaza et al [14]. GNEP solution concept in $n$-person non-cooperative games with common constraints gives infinitely many solutions. In order to select a unique equilibrium point among solutions in the game, we follow the approach proposed by Rosen in [15], which is known as normalized equilibrium, since it has suitable properties for decentralized scenarios.

In particular, we model our problem as a special subclass of the normalized equilibrium for non-cooperative game with coupled constraints, which we call Weakly Coupled Constrained Games (WCCG). Here the set of strategies $S_{f}$ available to a player $f$ depends on the strategies used by the other players in the game. This dependency appears in the common constraint to the agents but not in the players' utility function. We seek for a GNEP $s=\left(s_{1}, \ldots, s_{n}\right)$ where $s_{f} \in S_{f}$ for each player $f$ and where no player has any motivation to change its own strategy unilaterally to another one within the set $S_{f}$. Here, the WCCG is achieved when every HeNB realizes that, given the current strategy of the other players, any change in its own strategy to another one that satisfies the constraints would result in a decrease in the utility. We show for WCCG that if the utility function of each player is strictly concave in the strategies of that player, then the normalized Nash equilibrium is unique.

We propose players to be able to choose among different strictly concave utility functions, all depending on the same strategy set $S_{f}$. Players select the utility they want to maximize according to the metric they aim to optimize, i.e. throughput, Bit Error Rate (BER), packet delay. This introduces an interesting flexibility in the game, since players only have to fulfil the required common constraint independently of the parameter they are optimizing.

In the next section we begin with a motivating example. We then recall in Section III the WCCG equilibrium as a concept that allows a scalable equilibrium selection, and show its uniqueness for coupled constrain games. In Section IV we then compute the WCCG equilibrium for the examples we gave in Section III-B. Finally, in Section V we present our conclusions and future work.

\section{SCENARIO}

An OFDMA system operating in the downlink direction is considered, where the bandwidth $B$ is divided into $R$ Resource Blocks (RBs), with $B=R \cdot B_{\mathrm{RB}}$. A RB represents one basic time-frequency unit that occupies the bandwidth $B_{\mathrm{RB}}$ over time $T$. The considered scenario consists in two types of coexisting networks, i.e. eNB and HeNB transmitter-receiver couples. We consider $\mathcal{M}$ eNBs and $\mathcal{F}=n$ HeNBs systems. We refer to the eNB transmitter with $e N B_{m}, m \in \mathcal{M}$ and we represent the $f \in \mathcal{F} \mathrm{HeNB}$ as $\mathrm{HeNB}_{f}, f=1, \ldots, n$. Associated with each eNB and HeNB are $U^{\mathrm{M}}$ macro and $U^{\mathrm{F}}$ femto users, respectively. The multi-user resource assignment that distributes the $R$ RBs among the $U^{\mathrm{M}}$ macro and $U^{\mathrm{F}}$ femto users, is carried out by a proportional fair scheduler. We denote by $\mathbf{p}^{i}=\left(p_{1}^{i}, \ldots, p_{R}^{i}\right)$ the transmission power vector of BS $i$, with $p_{r}^{i}$ denoting the downlink transmission power of RB $r$. The maximum transmission power for HeNBs and eNBs are $P_{\text {max }}^{\mathrm{F}}$ and $P_{\text {max }}^{\mathrm{M}}$, with $P_{\text {max }}^{\mathrm{F}} \ll P_{\text {max }}^{\mathrm{M}}$, such that $\sum_{r=1}^{R} p_{r}^{m} \leq P_{\text {max }}^{\mathrm{M}}$ and $\sum_{r=1}^{R} p_{r}^{f} \leq P_{\max }^{\mathrm{F}}$.

We analyze the system performance in terms of Signal to Noise Ratio (SNR) and achieved data rate given in (bit/s). Assuming perfect synchronization in time and frequency, the SNR of user associated to $\mathrm{HeNB}_{f}$ who is allocated in RB $r$ amounts to:

$$
\gamma_{r}^{f}=\frac{p_{r}^{f} h_{r}^{f}}{\sigma^{2}}
$$

where $\sigma^{2}$ denotes the thermal noise power and $h_{r}^{m}$ represents the channel link between $e N B$ and $u^{m}, u^{m} \in U^{M}$. We represent the channel link between $\mathrm{HeNB}_{f}$ and $u^{f}, u^{f} \in U^{F}$, through $h_{r}^{f}$ and the interference link between $H e N B_{f}$ and $u^{m}$ through $\hat{h}_{r}^{f}$, as represented in Figure 1 .

We consider the system parameters and Path Loss (PL) models as proposed in [1] by 3GPP for both, HeNB and eNB systems. We model the link channels as presented in Table I. Here, $d$ and $d_{\text {indoor }}$ are the total and indoor distances between the eNB/HeNB and the femto and macrouser, respectively. The factor $0.7 d_{\text {indoor }}$ takes into account the penetration losses due to the walls inside the apartments. $W P_{\text {out }}=15 \mathrm{~dB}$ and $W P_{\text {in }}=5 \mathrm{~dB}$ are the penetration losses of the building 
TABLE I

PATH LOSS MODELS FOR URBAN DEPLOYMENT.

\begin{tabular}{|c|c|c|}
\hline eNB to macrouser & outdoors & $P L(\mathrm{~dB})=15.3+37.6 \log _{10} d$ \\
\hline \multirow{2}{*}{$\begin{array}{c}\text { HeNB to } \\
\text { macro/femtouser }\end{array}$} & femtouser & $P L(\mathrm{~dB})=38.46+20 \log _{10} d+0.7 d_{\text {indoor }}+w * W P_{\text {in }}$ \\
\cline { 2 - 3 } & macrouser & $P L(\mathrm{~dB})=\max \left(15.3+37.6 \log _{10} d, 38.46+20 \log _{10} d\right)+0.7 d_{\text {indoor }}+w * W P_{\text {in }}+W P_{\text {out }}$ \\
\hline
\end{tabular}

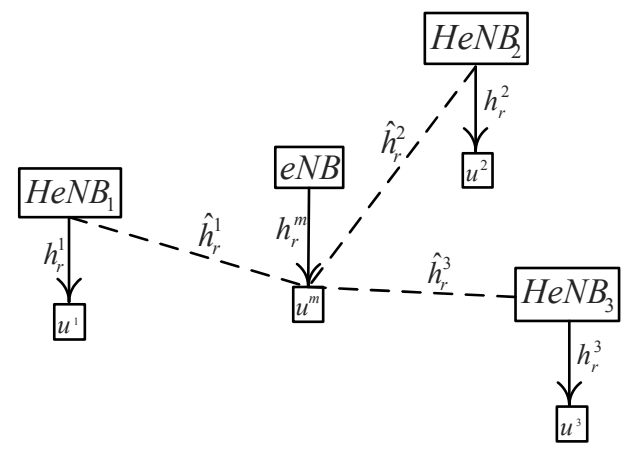

Fig. 1. Network components and channel links.

external walls and of the walls separating the apartments, respectively. Finally, $w_{p}$ represents the number of walls separating apartments.

\section{WEAKLY COUPLED CONSTRAINED GAMES}

In this section we introduce the concept of existence, uniqueness and selection of equilibrium for non-cooperative concave $n$-players games with coupled constraints. Then, we apply this theory to find the power allocation strategy in a HeNB.

$n$-person games are defined by $n$ players, where each player $f \in \mathcal{F}$ has its own set of pure strategies $S_{f}$ and a utility function $U^{f}$, which maps the $n$-tuple of pure strategies, one per player in the game, into a real number. Non-cooperative games are given by the incapacity of players in the game to share information, cooperate or share utilities. The existence of at least one equilibrium point in finite non-cooperative $n$-person games with or without orthogonal constraints was proven by Nash in [13]. Going a step further, the existence of equilibrium in games with coupled constraints, i.e. when the choice of strategies of a player depends on the strategies chosen by other agents, was extended by Arrow and Debreu in [3].

The uniqueness of equilibrium point for non-cooperative $n$ person strictly concave games was proven by Rosen in [15] through the normalized equilibrium concept. More in detail, Rosen considers problems where every player joint strategy, which is a point in the product space of the individual strategy spaces, lie in convex, closed and bounded region $C$ in the product space and that each player's utility function $U^{f}$ is a concave function in his own strategy [15]. To apply the normalized equilibrium concept, the game must fulfill an additional concavity condition, which is called diagonal strict concavity.

As mentioned in the introduction of this work, the problem we aim to solve perfectly fits in the coupled constrained noncooperative $n$-person game approach. We consider $n$ noncooperative players, i.e. the $n$ HeNBs in the system, where player $f \in \mathcal{F}$ and $f=1 \ldots n$, controls the variable $p_{r}^{f} \in \mathbb{R}^{d_{f}}$. Let $p_{r}$ be the $n$-dimensional vector of all players strategies with dimension $d=\sum_{f=1}^{n} d_{f}$ and $p_{r}^{-f}$ the $n-1$ vector formed by all players' strategies but $f$. Let $S \subset \mathbb{R}^{d}$ be a compact convex set.

Each player in the game has a utility function, $U^{f}: \mathbb{R}^{d_{f}} \rightarrow$ $\mathbb{R}$. An equilibrium in this game consists of a vector $p_{r}^{*} \in C$ such that for each player $f, U^{f}\left(p_{r}^{f}\right)$ attains its maximum over all $p_{r}^{f}$ for which $\left(p_{r}^{f}, p_{r}^{*}[-f]\right) \in C$. Here, $C$ is a convex compact set of constraints and $\left(p_{r}^{f}, p_{r}^{-f *}\right)$ is the policy obtained from $p_{r}^{*}$ by the strategies of all players, except for that player $f$ who uses $p_{r}^{f}$ instead of $p_{r}^{*}$. The maximization problem is then given by:

$$
\begin{aligned}
\underset{\left\{p_{r}^{f, F}\right\}}{\operatorname{maximize}} & U^{f}\left(p_{r}^{f}, p_{r}^{-f}\right) \\
\text { s.t. } & p_{r}^{f} \in \mathbf{p}^{f}\left(p_{r}^{-f}\right)
\end{aligned}
$$

In our problem, we consider the case where the $n$ HeNBs transmit in downlink direction to their associated users. We consider the setting in which the achievable utility of all HeNBs is given by the convex region $\nu$ defined by the set of constraints:

$$
\begin{gathered}
\sum_{f=1}^{n} \hat{h}_{r}^{f} p_{r}^{f} \leq I_{T h} \\
0 \leq p_{r}^{f} \leq P_{\max }^{F}
\end{gathered}
$$

where $I_{T h}$ is an interference constraint at macrouser $u^{m}$. Every player $f$ maximizes its own utility $U^{f}$, which is assumed to be a strictly concave increasing function of its strategy vector $\mathbf{p}^{f}$. Given the system model presented in Section II, we assume that the utility of a player depends only on its own strategy. We call such games WCCGs, where the interaction with other players occurs only through the constraints. This is a special case of normalized Nash equilibrium [15], where interactions between players are given by both, the utility function and the constraints.

The interference constraint, $I_{T h}$, at macrousers is a common constraint that all players strategies are required to satisfy, 
therefore, this places the game we are formulating in the category of coupled constraint games defined by Rosen [15]. In games with coupled constraints the choice of strategies of a player depends on the strategies chosen by other players.

The equilibrium notion of the given game is a special case of the so-called GNEP [8], where multiple players have to find a power allocation vector $p_{r}^{f}$ within $\nu$ such that no player $f$ can gain by deviating from $p_{r i}^{f}$ to $p_{r j}^{f}, i, j \in \mathbf{p}^{f}$, for which $\left(p_{r j}^{f}, p_{r}^{-f}\right) \in \nu$. When games with constraints are defined, it is not enough to consider the utility and constraints of each player, it is also required to specify how a player evaluates the fact that constraints of another player are satisfied or violated. We focus on studying the properties of the normalized equilibria introduced by Rosen in [15], since it properties are quiet appropriate for decentralized implementations.

Lemma 1: All HeNBs transmission powers, $p_{r}^{f}$, satisfying

$$
\sum_{f=1}^{n} \hat{h}_{r}^{f} p_{r}^{f}=I_{T h}
$$

are Nash equilibria and Pareto-efficient. Any other point is not an equilibrium.

Remark 1: The strategy of player $f$ in our game consists of choosing the i-th entry of the transmission power vector $\mathbf{p}^{f}$. A deviation of $f$ from a point in the achievable utility region $\nu$ to another one in that region affects only its own utility and not the other players one [2].

\section{A. Normalized Nash Equilibrium}

As mentioned before we propose that players choose the WCCG equilibrium, which is a special case of normalized Nash equilibrium, among the large number of Nash equilibriums. Normalized Nash equilibriums are based on pricing, which means that players' actions will have a price based on the constraints defining the game. We are interested in pricing mechanisms that induce equilibria strategies and that can be implemented in a scalar and decentralized way. We denote by $\lambda^{f}$ the transmitted power price of HeNB $f$ based on the total interference at $u^{m}$ and by $\bar{\lambda}$ the $n$-dimensional vector whose $f$-th entry is $\lambda^{f}$. Then, the resultant payoff of HeNB $f$ including the pricing would be:

$$
L_{\bar{\lambda}}^{f}\left(p_{r}^{f}\right)=U^{f}-\lambda^{f}\left(\sum_{f=1}^{n} \hat{h}_{r}^{f} p_{r}^{f}-I_{T h}\right)
$$

Consider now the following relaxed game. For each player $f$ find the $p_{r}^{f *} \in \nu$ given by:

$$
L_{\lambda}^{f}\left(p_{r}^{f *}\right) \geq L_{\lambda}^{f}\left(p_{r}^{f}, p_{r}^{-f *}\right)
$$

If the solution for $L_{\lambda}^{f}\left(p_{r}^{f *}\right)$ exists, it can be viewed as the Lagrangian solution corresponding to the constrained optimization problem faced by player $f$ when the other players play $p_{r}^{-f *}$. Based on the Karush-Kuhn-Tucker (KKT) conditions we know that there exists a vector $\bar{\lambda}$, whose entries are not necessarily equal, such that a power vector $p_{r}^{*}$ is an equilibrium in the original game only if for each player $f, p_{r}^{f *}$ maximizes
$L_{\lambda}^{f}\left(p_{r}^{f}, p_{r}^{-f *}\right)$. Thus, $\bar{\lambda}$ represents the non-scalable pricing, where $\lambda^{f}$ represents the price per transmitted power of player $f . \bar{\lambda}$ is non-scalable since the price depends on $f$.

Then, the $\mathrm{f}$-th component of $p_{r}^{*} \in \nu$ would be the solution of:

$$
\nabla U^{f}\left(p_{r}^{*}\right)-\sum_{f=1}^{n} \lambda^{f} \nabla g^{f}\left(p_{r}^{*}\right)=0
$$

for $\lambda^{f} \geq 0$. Here, $g^{f}$ represents the constraint function. We denote the solution as $p_{r}^{*}(\bar{\lambda})$.

Problem 1: Consider a constant $\lambda$ and let $\bar{\lambda}$ be a vector of dimension $n$ with all its entries $\lambda$. Then, we want to find $\lambda$ such that $p_{r}^{*}(\bar{\lambda})$ is an equilibrium of the original game.

An equilibrium associated with some constraint $\lambda$ that solves Problem 1 is a special case of the normalized equilibrium concept presented in [15].

Theorem 1: There exists a unique normalized equilibrium to the original problem associated with some $\lambda$ as defined in Problem 1 as long as there exists a strategy that satisfies the constraints.

Proof: Define $G$ to be the $n$-dimensional square matrix whose $G_{i j}$ entry is $\frac{\partial L_{f}(x)^{2}}{\partial x_{i} \partial x_{j}}$. All the off diagonal elements are zero since we assumed that the utility of a player does not depend on the actions of other players. The $n$ remaining diagonal elements of $G+G^{T}$ are strictly negative definite due to our assumption that the utility of player $f$ is strictly concave in the strategies of player $f$. We conclude that $G+G^{T}$ is strictly negative definite. The Proof then follows from [15, Theorem 6].

In our particular case we know that there exists at least one $n$-dimensional vector $p_{r}$, with all its elements $p_{r}^{f}=0$, which would satisfies the coupled constraint.

\section{B. HeNB interference management game}

We assume that players in this game can have different strictly concave utility functions satisfying the given restrictions. In the following we present the considered utility functions regarding throughput and performance.

1) Physical layer Shannon throughput:

$$
U^{f}=B \cdot \log _{2}\left(1+\gamma_{r}^{f}\right)
$$

2) Physical layer BER. OFDMA systems use Quadrature Phase-shift Keying (QPSK), 16 Quadrature Amplitude Modulation (QAM) and 64 QAM modulations. We propose to use a tight BER approximation for squared $M$-QAM for Additive White Gaussian Noise (AWGN) channels and ideal coherent phase detection [11] given by:

$$
\mathrm{BER}=0.2 \exp \left(-\frac{3 \gamma_{r}^{f}}{2(M-1)}\right)
$$

Then, the utility functions given any of the following modulations are:

- QPSK

$$
U^{f}=-0.2 \exp \left(-\frac{\gamma_{r}^{f}}{2}\right)
$$




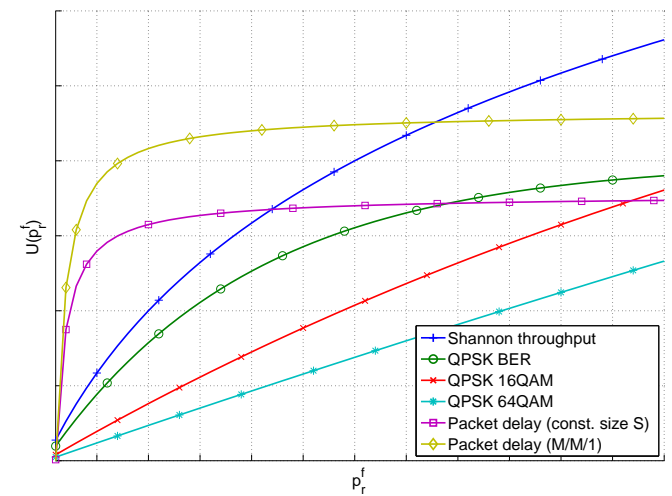

Fig. 2. Considered utilities functions at the HeNBs.

- 16 QAM

$$
U^{f}=-0.2 \exp \left(-\frac{\gamma_{r}^{f}}{10}\right)
$$

- 64 QAM

$$
U^{f}=-0.2 \exp \left(-\frac{\gamma_{r}^{f}}{42}\right)
$$

3) Packet delay $D$ in the link layer:

- Assuming files with constant size $S$ and link transmission throughput $\theta\left(p_{r}^{f}\right)$, then $U^{f}=-D$ where $D=\frac{S}{\theta\left(p_{r}^{f}\right)}$, then:

$$
U^{f}=-\frac{S}{B \cdot \log _{2}\left(1+\gamma_{r}^{f}\right)}
$$

- Assuming an M/M/1 queuing system, where files have an exponentially distributed size with rate $\eta$ and arrive according to a Poisson process with arrival rate $\rho$, the packet delay is given by $D=\frac{1}{\eta-\rho}$, and then the utility function is:

$$
U^{f}=-\frac{1}{B \cdot \log _{2}\left(1+\gamma_{r}^{f}\right)-\rho}
$$

In Figure 2 the above introduced utility functions are represented. Notice that the aim of this figure is only to show the different functions behavior in order to demonstrate their strictly concave behavior.

\section{REsults}

In what follows we present the solution of equation (6) considering the different proposed utility functions. The given solutions represent the closed solution $p_{r}^{f *}$ for agent $f$. For the throughput utility function given in equation (7) we obtained.

$$
\begin{gathered}
B \cdot \log _{2}\left(1+\frac{p_{r}^{f} h_{r}^{f}}{\sigma^{2}}\right)-\lambda^{f}\left(\sum_{f=1}^{n} \hat{h}_{r}^{f} p_{r}^{f}-I_{T h}\right)=0 \\
p_{r}^{f^{*}}=\left(\frac{B}{\ln (2) \lambda^{f} \hat{h}_{r}^{f}}-\frac{\sigma^{2}}{h_{r}^{f}}\right)^{+}
\end{gathered}
$$

Then, the common price for the players given by the Lagrange multiplier is:

$$
\lambda^{f}=\frac{B h_{r}^{f}}{\ln (2)\left(\hat{h}_{r}^{f} \sigma^{2}+I_{T h} h_{r}^{f}\right)}
$$

For the BER utility function for the QPSK modulation given in equation (8) we obtained.

$$
p_{r}^{f^{*}}=\left(-\frac{2 \sigma^{2}}{h_{r}^{f}} \ln \left(\frac{10 \sigma^{2} \lambda^{f} \hat{h}_{r}^{f}}{h_{r}^{f}}\right)\right)^{+}
$$

The common price for the players given by the Lagrange multiplier is:

$$
\lambda^{f}=\frac{h_{r}^{f}}{10 \sigma^{2} \hat{h}_{r}^{f}} \exp \left(-\frac{I_{T h} h_{r}^{f}}{2 \sigma^{2} \hat{h}_{r}^{f}}\right)
$$

For the BER utility function for the 16 QAM modulation given in equation (9) we obtained.

$$
p_{r}^{f^{*}}=\left(-\frac{10 \sigma^{2}}{h_{r}^{f}} \ln \left(\frac{50 \sigma^{2} \lambda^{f} \hat{h}_{r}^{f}}{h_{r}^{f}}\right)\right)^{+}
$$

And the common price for the players given by the Lagrange multiplier is:

$$
\lambda^{f}=\frac{h_{r}^{f}}{50 \sigma^{2} \hat{h}_{r}^{f}} \exp \left(-\frac{I_{T h h_{r}^{f}}}{10 \sigma^{2} \hat{h}_{r}^{f}}\right)
$$

For the BER utility function for the 64 QAM modulation given in equation (10) we obtained.

$$
p_{r}^{f^{*}}=\left(-\frac{42 \sigma^{2}}{h_{r}^{f}} \ln \left(\frac{210 \sigma^{2} \lambda^{f} \hat{h}_{r}^{f}}{h_{r}^{f}}\right)\right)^{+}
$$

In this case the common price for the players given by the Lagrange multiplier is:

$$
\lambda^{f}=\frac{h_{r}^{f}}{210 \sigma^{2} \hat{h}_{r}^{f}} \exp \left(-\frac{I_{T h} h_{r}^{f}}{42 \sigma^{2} \hat{h}_{r}^{f}}\right)
$$

The packet delay utility function (11) is highly complex to solve. We shall therefore restrict utility to low SNR regime, which means that the approximation $\ln (1+x) \simeq x$ holds. This regime is of interest since HeNBs are low-power cellular BSs. Specifically, for the packet delay utility function for files with constant size given in equation (11), solution is given by:

$$
\begin{gathered}
-\frac{S}{B \cdot \gamma_{r}^{f}}-\lambda^{f}\left(\sum_{f=1}^{n} \hat{h}_{r}^{f} p_{r}^{f}-I_{T h}\right)=0 \\
p_{r}^{f^{*}}=\frac{\sqrt{S B \sigma^{2} \lambda^{f} \hat{h}_{r}^{f} h_{r}^{f}}}{\lambda^{f} \hat{h}_{r}^{f} h_{r}^{f}}
\end{gathered}
$$

And the common price for the players given by the Lagrange multiplier is:

$$
\lambda^{f}=\frac{S \sigma^{2} \hat{h}_{r}^{f}}{B h_{r}^{f} I_{T h}^{2}}
$$


For the packet delay utility function for the case of files with exponentially distributed size and Poisson arrival given in equation (12) solution is given by:

$$
\begin{gathered}
-\frac{1}{B \gamma_{r}^{f}-\lambda}-\lambda^{f}\left(\sum_{f=1}^{n} \hat{h}_{r}^{f} p_{r}^{f}-I_{T h}\right)=0 \\
p_{r}^{f^{*}}=\left(\frac{\lambda^{f} \hat{h}_{r}^{f} \rho \sigma^{2} \pm \sqrt{B \sigma^{2} \lambda^{f} \hat{h}_{r}^{f} h_{r}^{f}}}{B \lambda^{f} \hat{h}_{r}^{f} h_{r}^{f}}\right)^{+}
\end{gathered}
$$

The common price for the players given by the Lagrange multiplier is:

$$
\lambda^{f}=\frac{B \hat{h}_{r}^{f} \sigma^{2} h_{r}^{f}}{\left(\hat{h}_{r}^{f} \sigma^{2} \rho-B I_{T h} h_{r}^{f}\right)^{2}}
$$

\section{CONCLUSIONS}

In the present work we have modeled a multi HeNB scenario in coexistence with a eNB system as a non-cooperative $n$ person game. Given the common nature of the interference from the multiple HeNBs at eNB users, which is considered as a restriction in the game, we proposed a special form GNEP equilibrium concept for coupled constraint games, the WCCG. We found the normalized equilibrium among the infinitely many equilibriums of the given coupled game in a closed form for multiple concave utility functions that HeNBs can choose depending on the parameter they aim to maximize, i.e. throughput, BER, packet delay. As future work we aim to obtain further simulation results for the proposed game model.

\section{ACKNOWLEDGMENTS}

This work has been partially funded by the Spanish Research Council under SOFOCLES grant (TEC2010-21100), by European Union under ICT project ICT-4-248523 BeFEMTO, ACROPOLIS-NoE (ICT-2009.1.1), COST Action IC0902 and by the Spanish Ministry of Education (FPU grant AP20094993).

\section{REFERENCES}

[1] 3GPP. 3GPP TSG RAN WG4 (radio) meeting 51: Simulation assumptions and parameters for FDD HeNB RF requirements. Technical report, 4-8 May 2009.

[2] E. Altman, K. Avrachenkov, L. Cottatellucci, M. Debbah, G. He, and A. Suarez. Operating point selection in multiple access rate regions. In Proc. of the 21 st International Teletraffic Congress (ITC 21), 2009.

[3] K. J. Arrow and G. Debreu. Existence of an Equilibrium for a Competitive Economy. Econometrica, 22(3):265290, 1954.

[4] M. Bennis and S. M. Perlaza. Decentralized cross-tier interference mitigation in cognitive femtocell networks.
In Proceedings of IEEE International Conference on Communications, ICC 2011, Kyoto, Japan, 5-9 June, 2011.

[5] V. Chandrasekhar and J. G. Andrews. Uplink capacity and interference avoidance for two-tier femtocell networks. IEEE Trans. Wireless Communications, 8(7):3498-3509, July 2009.

[6] V. Chandrasekhar, J. G. Andrews, and A. Gatherer. Femtocell networks: a survey. Communications Magazine, IEEE, 46(9):59-67, Sept. 2008.

[7] R. Combes, Z. A. M. Haddad, and E. Altman. Selfoptimizing strategies for interference coordination in OFDMA networks. In Proc. of the 2011 IEEE International Conference on Communications Workshops (ICC), Kyoto, Japan, 5-9 June 2011.

[8] F. Facchinei and C. Kanzow. Generalized nash equilibrium problems. Annals OR, 175(1):177-211, 2010.

[9] S. C. Forum. Interference management in OFDMA femtocells. http://www.smallcellforum.org/.

[10] L. Giupponi and C. Ibars. Distributed interference control in OFDMA-based femtocells. In Proceedings of the IEEE 21st International Symposium on Personal, Indoor and Mobile Radio Communications, PIMRC 2010, 26-29 September 2010, Istanbul, Turkey, pages 1201-1206.

[11] A. J. Goldsmith, S. ghee Chua, and A. Member. Variablerate variable-power MQAM for fading channels. IEEE Trans. Commun, 45:1218-1230, 1997.

[12] D. López-Pérez, A. Valcarce, G. de la Roche, and J. Zhang. OFDMA femtocells: A roadmap on interference avoidance. IEEE Communications Magizine, 47(9):41-48, Sept. 2009.

[13] J. Nash. Non-Cooperative Games. The Annals of Mathematics, 54(2):286-295, Sep. 1951.

[14] S. M. Perlaza, H. Tembine, S. Lasaulce, and M. Debbah. Satisfaction equilibrium: A general framework for qos provisioning in self-configuring networks. In GLOBECOM, pages 1-5, 2010.

[15] J. Rosen. Existence and uniqueness of equilibrium points for concave N-person games. Econometrica, 33(3):520534, 1965.

[16] S. Uygungelen, Z. Bharucha, and G. Auer. Decentralized interference coordination via autonomous component carrier assignment. In Workshops Proceedings of the Global Communications Conference, GLOBECOM 2011, 5-9 December 2011, Houston, Texas, USA, pages 219224, 2011.

[17] J. Zhang and G. de la Roche. Femtocells: Technologies and Deployment. John Wiley \& Sons Ltd., New York, NY, USA, 2010. 